\title{
PROPOSTA DE MÉTODO DE REDE GNSS POR PPP E ANÁLISE DE CONFIABILIDADE
}

\author{
Proposed Methodology for GNSS Network by PPP and analysis of Reliability
}

\author{
Carolina Collischonn ${ }^{1,2}$ \\ Marcelo Tomio Matsuoka 1,3 \\ 1Programa de Pós-Graduação em Sensoriamento Remoto- Universidade Federal do Rio Grande do Sul \\ - Porto Alegre/RS.Email: carol.collischonn@gmail.com. \\ 2Instituto Federal de Santa Catarina/IFSC - Florianópolis/SC \\ ${ }^{3}$ Instituto de Geografia - Engenharia de Agrimensura e Cartográfica - Universidade Federal de \\ Uberlândia - Campus Monte Carmelo - Monte Carmelo/MG
}

\section{Resumo:}

Embora o método de posicionamento GNSS tradicionalmente utilizado em redes geodésicas seja o relativo. O nível de acurácia obtido com o método de Posicionamento por Ponto Preciso (PPP) abre uma nova perspectiva. No PPP são utilizados dados de apenas um receptor GNSS e é fundamental o uso de efemérides e correções dos relógios dos satélites, ambas com alta precisão. Neste artigo é apresentada uma metodologia de desenvolvimento de rede utilizando dados GNSS processados pelo método de PPP e a verificação da sua potencialidade em aplicações geodésicas. Os dados utilizados são de estações GNSS pertencentes à RBMC/IBGE. O serviço de processamento de PPP utilizado é o fornecido pelo IBGE. A partir dos resultados foram feitas análises para verificar a aplicabilidade da metodologia descrita em rede com dados GNSS de 24, 6 e 4 horas de rastreio. Após o ajustamento, os testes global e data snooping foram aplicados e também foi analisada a confiabilidade da rede com o objetivo de avaliar o método proposto, além de verificar a influência do tempo de rastreio nos resultados.

Palavras-chave: rede GNSS, PPP, confiabilidade.

\section{Abstract:}

The relative GNSS positioning method is traditionally used in geodetic networks. Other GNSS positioning method is the Precise Point Positioning (PPP). In the PPP data from only one GNSS receiver are used and the use of precise ephemeris and satellite clocks corrections are essential. This paper presents a methodology for network development using GNSS data processed by the PPP method and checking their potential in geodetic applications. GNSS data from some RBMC/IBGE stations were used. The PPP processing service used is the provided by IBGE. From the PPP result analyzes were performed to verify the applicability of the methodology 
described in GNSS network with data by 24, 6 and 4 hours of tracking. After GNSS data adjustment, the global and data snooping testing were applied and also analyzes of the network reliability in order to evaluate the proposed methodology and to verify the influence of tracking time in the results.

Keywords: GNSS Network, PPP, reliability.

\section{Introdução}

Tradicionalmente o método de posicionamento GNSS (Global Navigation Satellite System) utilizado em redes geodésicas é o relativo. No posicionamento relativo utilizam-se, em geral, as duplas diferenças (DD) como observáveis fundamentais e dois ou mais receptores envolvidos rastreiam, simultaneamente, pelo menos dois satélites comuns. Nesse método, são estimadas as componentes da linha-base $(\Delta \mathrm{X}, \Delta \mathrm{Y}$ e $\Delta \mathrm{Z})$ entre duas ou mais estações. Uma linha-base envolve duas estações, podendo ser uma supostamente conhecida e outra a determinar, as duas estações conhecidas, ou ainda, ambas as estações desconhecidas, fazendo parte de uma rede. As componentes que compõem a linha-base são estimadas e, ao serem acrescentadas às coordenadas da estação-base ou de referência (estação com coordenadas conhecidas), proporcionam as coordenadas da estação desejada (Monico, 2008). As componentes das linhas-base são estimadas, podendo ser em softwares comerciais, de forma individual ou levando em conta a correlação entre as várias linhas-base simultâneas de cada sessão. Na maioria dos casos, a partir das soluções individuais de cada linha-base, proporcionada pelo processamento do método de posicionamento relativo, bem como injunções das coordenadas dos vértices conhecidos, é feito o ajustamento da rede para a obtenção das coordenadas e precisões dos vértices desconhecidos.

Outro método de posicionamento GNSS existente é o Posicionamento por Ponto Preciso (PPP). Esse método é empregado desde a década de 1990, primeiramente limitando-se a aplicações científicas. Nos últimos anos o PPP passou a ser empregado mais amplamente, também no Brasil, principalmente devido ao surgimento de serviços on-line gratuitos de processamento com facilidade de acesso e simplicidade de uso (Matsuoka et. al., 2009). No PPP utilizam-se os dados de apenas um receptor GNSS (Leick, 2004) e é fundamental o uso de efemérides e correções dos relógios dos satélites, ambos com alta precisão (Monico, 2008), Marques et al. (2014) e Souza et al. (2014). O método PPP a partir de serviços on-line tem sido estudado nos últimos anos, podendo-se citar os seguintes trabalhos: Matsuoka et al.(2009); Klein et al. (2010), Perdigão et al. (2010), Ocalan et al. (2013). Alguns trabalhos internacionais publicados sobre o PPP e avaliações dos serviços oferecidos podem ser citados, por exemplo: Zumberge et al (1997), Kouba, J.; Héroux, P. (2001), Gao, Y e Shen, X (2002) e El-Mowafy (2011).

O PPP fornece as coordenadas de estações de forma isolada, sem conexões com outras estações, ou seja, sem a formação de uma linha-base da forma tradicional como a citada. Dessa forma, os resultados obtidos apenas com o PPP possuem a desvantagem de não se valerem de análises como as aplicadas no caso de redes GNSS, tais como: análise de fechamento de circuitos, aplicação de testes estatísticos em rede e análises de confiabilidade.

Neste trabalho é apresentada uma proposta de método de desenvolvimento de rede utilizando resultados de estações GNSS processados por PPP. Além da apresentação do método são 
realizados experimentos aplicando a metodologia descrita. Os experimentos são realizados utilizando dados GNSS com diferentes tempos de rastreio e as análises dos resultados são realizadas a fim de verificar a potencialidade do uso do método em aplicações geodésicas.

\section{Fundamentos do PPP}

De acordo com Monico (2008), no PPP são utilizadas as observáveis pseudodistância e/ou fase da onda portadora, coletadas por receptores de simples ou dupla-frequência, efemérides precisas e correções dos relógios dos satélites precisas. Segundo Leick (2004) o PPP refere-se ao posicionamento com GNSS utilizando dados de apenas um receptor, com precisão em torno do centímetro quando se considera o modo estático e um longo período de coleta de dados até a precisão da ordem de alguns decímetros, quando utilizado o modo cinemático. Nesses casos, para atingir este nível de qualidade, deve-se considerar como observável no processamento a combinação linear livre da ionosfera (ion-free) para a fase da onda portadora e/ou código (Leick, 2004; Hofmann-Wellenhof et al., 2008), o que obviamente implica no uso de receptor de duplafrequência. Quando utilizados receptores de simples frequência, devem-se minimizar os efeitos da ionosfera com emprego de algum modelo da ionosfera disponível (Matsuoka et. al., 2009 e Rocha et al., 2014).

Ainda segundo Monico (2008), nas efemérides precisas, ou pós-processadas, a órbita (posição) e o erro do relógio (tempo) dos satélites são determinados com alta precisão por algum serviço e disponibilizados por algum meio de comunicação, como, por exemplo, a internet. Essas informações têm sido produzidas e disponibilizadas pelo IGS (International GNSS Service) e centros associados, sem nenhum custo. O IGS produz três tipos de efemérides e correções para o relógio dos satélites denominadas de efemérides IGS, IGR e IGU, cuja descrição detalhada pode ser obtida em https://igs.org/products.

A qualidade das coordenadas estimadas no PPP está relacionada principalmente aos erros considerados no ajustamento de observações, cuja remoção parcial ou completa dos seus efeitos é realizada através dos modelos matemáticos (Rosa, 2008). Quando se utilizam dados de receptores de dupla frequência, tanto de pseudodistância quanto de fase, os modelos matemáticos envolvidos no PPP que descrevem as observáveis depois de realizada a combinação linear ionfree, são normalmente dados por (Leick, 2004; Monico, 2008):

$$
\begin{aligned}
& \mathrm{PD}_{\mathrm{r}(\mathrm{IF})}^{\mathrm{s}}=\rho_{\mathrm{r}}^{\mathrm{s}}+\mathrm{c}\left(\mathrm{dt}_{\mathrm{r}}-\mathrm{dt}^{\mathrm{s}}\right)+\mathrm{T}_{\mathrm{r} 0}^{\mathrm{s}}+\mathrm{dT}_{\mathrm{z}} \mathrm{m}(\mathrm{E}) \\
& \varphi_{\mathrm{r}(\mathrm{IF})}^{\mathrm{s}}=\frac{\mathrm{f}_{1}}{\mathrm{c}} \rho_{\mathrm{r}}^{\mathrm{s}}+\mathrm{f}_{1}\left(\mathrm{dt}_{\mathrm{r}}-\mathrm{dt}^{\mathrm{s}}\right)+\mathrm{N}_{\mathrm{IF}}+\frac{\mathrm{f}_{1}}{\mathrm{c}} \mathrm{T}_{\mathrm{r} 0}^{\mathrm{s}}+\frac{\mathrm{f}_{1}}{\mathrm{c}} \mathrm{dT}_{\mathrm{z}} \mathrm{m}(\mathrm{E})
\end{aligned}
$$

Em que:

$$
\begin{aligned}
& \mathrm{PD}_{\mathrm{r}(\mathrm{IF})}^{\mathrm{s}}=\text { pseudodistância resultante da combinação linear ion-free; } \\
& \varphi_{\mathrm{r}(\mathrm{IF})=\text { fase obtida da combinação linear ion-free; }}^{\mathrm{s}} \\
& \rho_{\mathrm{r}}^{\mathrm{s}}=\text { distância geométrica entre o centro de fase da antena do satélite e do receptor; } \\
& \mathrm{dt}_{\mathrm{r}}=\text { erro do relógio do receptor; }
\end{aligned}
$$


$\mathrm{dt}^{\mathrm{s}}=$ erro do relógio do satélite;

$\mathrm{N}_{\mathrm{IF}}=$ ambiguidade da observável ion-free;

$\mathrm{T}_{\mathrm{r} 0}^{\mathrm{s}}=$ atraso troposférico aproximado por algum modelo disponível;

$\mathrm{dT}_{\mathrm{z}}=$ atraso zenital troposférico residual a ser estimado no modelo;

$\mathrm{m}(\mathrm{E})$ = função de mapeamento em função do ângulo de elevação E do satélite;

$\mathrm{c}=$ velocidade da luz no vácuo; e

$\mathrm{f}_{1}=$ é a frequência da observável ion-free (igual à da portadora L1).

No modelo matemático apresentado os demais erros sistemáticos foram negligenciados. Considerando a Equação (1), pode-se observar que os parâmetros (incógnitas) a serem estimados envolvem as coordenadas da estação (presentes no termo $\rho_{\mathrm{r}}^{\mathrm{s}}$ ), o erro do relógio do receptor (dt $)$, a correção residual da troposfera $\left(\mathrm{dT}_{\mathrm{z}}\right)$ e o vetor de ambiguidades da ion-free $\left(\mathrm{N}_{\mathrm{IF}}\right)$. O erro do relógio do satélite $\left(\mathrm{dt}^{\mathrm{S}}\right)$ é injuncionado pelo valor disponibilizado pelo IGS ou por qualquer outro centro de análise. $\mathrm{O}$ valor do atraso troposférico (direção receptor-satélite) aproximado $\left(\mathrm{T}_{\mathrm{r} 0}^{\mathrm{s}}\right)$ é obtido pelo uso de algum modelo disponível, como por exemplo, Hopfield ou Saastamoinen (Monico, 2008).

Outros erros, como o relacionado ao centro de fase da antena dos satélites e do receptor também devem ser tratados no PPP. Adicionalmente, os erros relacionados a marés terrestres, carga dos oceanos, entre outros, podem também ser considerados. Uma descrição detalhada dos erros envolvidos pode ser encontrada em Witchayangkoon (2000), Leick (2004), Faustino (2006), Hofmann-Wellenhofet al. (2008) e Monico (2008).

Serviços de PPP são disponibilizados de forma gratuita e de processamento on-line, tais como o serviço de PPP do JPL (Jet Propulsion Laboratory), da UNB (Universityof New Brunswick), do NRCan (Natural Resources Canada) e, no Brasil, o serviço do IBGE, que é utilizado nesse artigo, entre outros. O IBGE-PPP é um serviço on-line para o pós-processamento de dados GPS pelo PPP. Os dados podem ser no modo estático ou cinemático, de receptores de simples ou de dupla frequência. O sistema aceita arquivos nos formatos RINEX ou Hatanaka, caso seja mais de um arquivo, devem ser comprimidos. Além disso, é necessário o modelo da antena do receptor utilizado no levantamento, conforme identificação adotada pelo IGS (International GNSS Service) e o valor da altura da antena, em metros, referidos ao plano de referência da antena. Os resultados são informados através de e-mail fornecido pelo usuário quando este submete os dados para processamento. Este serviço de posicionamento faz uso do aplicativo de processamento CSRS-PPP (Canadian Spatial Reference System - PPP) desenvolvido pelo NRCan. Para mais informações sobre o PPP ver em Zumberge et al (1997), Leick (2004) e Monico, 2008, assim como para mais especificações do serviço IBGE-PPP, consultar IBGE (2013).

\section{Método de formação de rede GNSS por PPP}

O método proposto para o desenvolvimento de rede, utilizando coordenadas estimadas através do método de PPP, consiste em, primeiramente, planejar a localização dos vértices da rede e as suas linhas-base. A rede deve ser planejada de modo que se formem linhas-base independentes em Bol. Ciênc. Geod., sec. Artigos, Curitiba, v. 22, no3, p.453-471, jul-set, 2016. 
sessões de rastreio GNSS. Por linhas-base independentes entende-se que nenhuma delas é combinação linear das outras. Na prática, para se garantir linhas-base independentes, pode-se, por exemplo, tendo como base um circuito fechado de três linhas-base, obter os dados dos pontos de duas linhas-base em um mesmo período de rastreio e a dos pontos da terceira linha-base em um período diferente.

Planejada a rede, o levantamento e processamento dos dados GNSS são realizados pelo método de PPP. Esse processamento pode ser realizado em serviços disponibilizados de forma gratuita e de processamento on-line (alguns já supracitados). Com o processamento dos dados GNSS pelo método do PPP são obtidos, dentre outras informações, para cada ponto levantado, as coordenadas cartesianas geocêntricas (X, Y, Z), os desvios-padrão e correlações entre as suas coordenadas. A MVC das coordenadas é obtida a partir dos valores de desvios-padrão de cada coordenada estimar e correlações associadas entre as coordenadas.

Para formar a Matriz Variância Covariância (MVC) das coordenadas de cada ponto, os resultados de desvios-padrão e correlação dessas coordenadas são convertidos em variância e covariância. A MVC de cada ponto processado por PPP é uma matriz de dimensão $3 \times 3$ (três linhas e três colunas).

Para a formação da rede, para posterior ajuste, é necessário obter as componentes de cada linhabase $(\Delta \mathrm{X}, \Delta \mathrm{Y}$ e $\Delta \mathrm{Z})$ e sua respectiva MVC. As linhas-base entre os vértices da rede são determinadas a partir das diferenças entre as coordenadas $\mathrm{X}, \mathrm{Y}$ e $\mathrm{Z}$, dos vértices, advindos do processamento PPP. Assim, considerando dois vértices $\mathrm{i}$ e $\mathrm{j}$ tem-se respectivamente, as coordenadas $\mathrm{Xi}, \mathrm{Yi}, \mathrm{Zi}$ e $\mathrm{Xj}, \mathrm{Yj}, \mathrm{Zj}$ obtidas por PPP e, consequentemente as componentes das linhas-base são:

$$
\begin{aligned}
\Delta X_{i j} & =X_{j}-X_{j} \\
\Delta Y_{i j} & =Y_{j}-Y_{j} \\
\Delta Z_{i j} & =Z_{j}-Z_{i}
\end{aligned}
$$

A MVC de cada linha-base é obtida por meio da aplicação da Lei de Propagação de Variância e Covariância (Gemael, 1994). Para o exemplo dos vértices i e j, a partir da MVC $\mathbb{E}_{\mathbb{P}} \mathbb{P}_{\mathbb{R}}$, bloco diagonal, contendo as variâncias e covariâncias dos dois vértices pertencentes à linha-base, de dimensão 6x6, e da matriz dos coeficientes lineares $(\mathrm{G})$, de dimensão $3 \times 6$, é obtida a MVC das

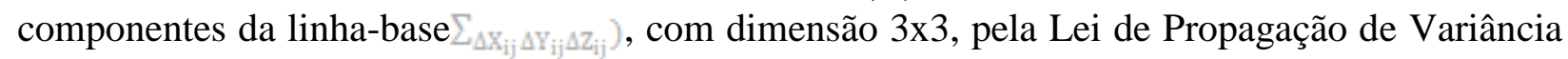
e Covariância:

$$
\sum_{\Delta X_{n} \Delta Y_{n} \Delta z_{n}}=G \sum_{P P P(i j)} G^{T}
$$

sendo:

$$
\sum_{\left(\Delta X_{i j} \Delta Y_{y j} \Delta z_{i j)}\right.}=\left[\begin{array}{ccc}
\sigma_{\Delta X i j}^{2} & \sigma_{\Delta X, \Delta Y} & \sigma_{\Delta X, \Delta z} \\
\sigma_{\Delta Y, \Delta X} & \sigma_{\Delta Y, j}^{2} & \sigma_{\Delta Y, \Delta z} \\
\sigma_{\Delta z, \Delta X} & \sigma_{\Delta Z, \Delta Y} & \sigma_{\Delta Z i j}^{2}
\end{array}\right]
$$




$$
\begin{gathered}
G=\left[\begin{array}{cccccc}
-1 & 0 & 0 & 1 & 0 & 0 \\
0 & -1 & 0 & 0 & 1 & 0 \\
0 & 0 & -1 & 0 & 0 & 1
\end{array}\right] \\
\sum_{P P P(i j)}=\left[\begin{array}{cccccc}
\sigma_{X i}^{2} & \sigma_{X Y} & \sigma_{X z} & 0 & 0 & 0 \\
\sigma_{Y X} & \sigma_{Y i}^{2} & \sigma_{Y Z} & 0 & 0 & 0 \\
\sigma_{Z X} & \sigma_{Z Y} & \sigma_{Z i}^{2} & 0 & 0 & 0 \\
0 & 0 & 0 & \sigma_{X j}^{2} & \sigma_{X Y} & \sigma_{X z} \\
0 & 0 & 0 & \sigma_{Y X} & \sigma_{Y j}^{2} & \sigma_{Y Z} \\
0 & 0 & 0 & \sigma_{Z X} & \sigma_{Z Y} & \sigma_{Z j}^{2}
\end{array}\right]
\end{gathered}
$$

$\mathrm{Na}$ Equação 4, $\sigma_{\Delta x i \mathfrak{j}}^{2}, \sigma_{\Delta x i j}^{2}$ e $\sigma_{\Delta z i j}^{2}$ são as variâncias das componentes das linhas-base entre os pontos i e j e demais elementos são as covariâncias associadas. Em (5), $\sigma_{\mathrm{Xi}}^{2}, \sigma_{\mathrm{Yi}}^{2} \sigma_{\mathrm{Zi}}^{2}, \sigma_{\mathrm{Xj}}^{2}, \sigma_{\mathrm{Yj}}^{2}, \sigma_{\mathrm{Zj}}^{2}$ são as variâncias dos pontos i e j e demais elementos as covariâncias, todas advindas do resultado do PPP dos vértices i e j.

Para cada linha-base da rede se procede da mesma forma descrita anteriormente. Com todas as linhas-base da rede e as suas respectivas MVC obtidas, realiza-se o ajustamento da rede, considerando também as injunções necessárias. A solução do ajustamento se dá pelo Método dos Mínimos Quadrados (MMQ) (Gemael, 1994). Por meio do método paramétrico do MMQ é possível estimar o vetor dos parâmetros ajustados, bem como a sua MVC. Mais detalhes quanto ao MMQ, consultar, por exemplo, Gemael (1994), Leick (2004) e Ghilani e Wolf (2006). Especificamente sobre o tema ajustamento por MMQ de rede GNSS pode-se consultar, por exemplo, Monico (2008) e Ghilani e Wolf (2006).

\section{Elementos utilizados para a análise da rede GNSS}

Após o ajustamento pelo MMQ ter sido realizado, dois dos testes estatísticos mais utilizados para a detecção e a identificação de erros (outliers) nas observações, são aplicados, respectivamente, o teste global do ajustamento e o procedimento de teste data snooping (DS), ambos propostos por Baarda (1968). O teste global do ajustamento é aplicado para a detecção de erros no conjunto de observações e/ou no modelo matemático, e mais detalhes sobre o mesmo podem ser obtidos em Baarda (1968), Kavouras (1982), Gemael (1994) e Teunissen (2006). O procedimento DS é aplicado para identificação de observações com erro grosseiro, no qual as observações são consideradas individualmente, sendo assim, o procedimento é repetido até que todas as observações que contenham erros grosseiros sejam identificadas e retiradas do conjunto de observações e o ajustamento seja aceito no teste global. Mais detalhes sobre o teste DS são encontrados em Baarda (1968), Teunissen (2006) e Klein (2012). O poder do teste global e do DS deve ser o mesmo. Entretanto, como os graus de liberdade destes dois testes são diferentes, o nível de significância do teste global deve ser obtido em função do nível de significância que foi arbitrado para o DS. Uma discussão mais detalhada do tema pode ser obtida em Baarda (1968), Kavouras (1982), Machado e Monico (2004), Teunissen (2006) e Klein (2012). 
A teoria convencional de confiabilidade é baseada no teste DS, assim assume-se que apenas uma observação está contaminada por erros por vez. A confiabilidade interna de uma observação se refere ao módulo do menor erro detectável (minimal detectable bias - MDB) desta observação, segundo os níveis de probabilidade que foram assumidos para o teste estatístico de identificação de erros (DS). Desta forma, para cada observação, o módulo do seu menor erro detectável (MDB) depende do parâmetro de não centralidade do modelo, que expressa a separação entre a hipótese nula (ausência de erros grosseiros na observação) e a hipótese alternativa considerada (presença de erros grosseiros na observação).Como a magnitude dos erros que contaminam as observações é desconhecida na prática, o verdadeiro valor para o parâmetro de não centralidade do modelo não pode ser determinado, mas pode ser obtido em função dos níveis de probabilidade assumidos e do número de graus de liberdade do teste estatístico em questão (Teunissen, 2006).

A confiabilidade externa, por sua vez, expressa a influência de um possível erro não detectado em uma observação, nos resultados finais do ajustamento, ou seja, no vetor dos parâmetros ajustados (Oliveira e Dalmolin, 2008). Para cada observação, a confiabilidade externa pode ser obtida substituindo o vetor das observações pelo modelo de erro, considerando o MDB, no estimador por mínimos quadrados dos parâmetros. Outra medida de confiabilidade é a razão tendência-ruído (bias to noise-ratio- BNR) da confiabilidade externa (ver, por exemplo, Klein (2012) e Teunissen (2006)) que é uma medida de confiabilidade externa que resulta em um único escalar para cada observação, facilitando as análises. O BNR é adimensional e quanto maior o BNR menor é a confiabilidade da rede.

Além dessas medidas tem-se o número de redundância local de cada observação, que corresponde à contribuição de cada observação para a redundância total do sistema de equações (Kavouras, 1982), e indica a fração de um possível erro grosseiro na observação que é diretamente refletida no resíduo desta observação, obtido com o ajustamento pelo MMQ. Analogamente, o número de absorção local é o complemento do número de redundância e indica a fração de um possível erro grosseiro na observação que é absorvido nos parâmetros incógnitos, não sendo refletido no respectivo resíduo desta observação. Para a identificação de um erro em uma observação é desejável um alto valor para o número de redundância, pois os testes para a identificação de erros apresentados dependem da magnitude dos resíduos ajustados. Mais detalhes sobre a confiabilidade convencional e os números de confiabilidade podem ser obtidos, por exemplo, em Teunissen (2006) e Klein (2012).

A rede por PPP realizada neste estudo, com base no método proposto, é avaliada nesse artigo com base no teste global, no DS, na confiabilidade interna e externa, no número de redundância e no BNR, todos descritos anteriormente. A formulação matemática envolvida em cada um deles não é descrita neste artigo por ser um assunto já consagrado na área da Geodésia e podem ser encontrados mais detalhes nos trabalhos que foram citados.

\section{Experimentos}

No presente artigo a proposta de desenvolvimento de rede por PPP é aplicada em uma rede GNSS constituída por dados das estações ILHA (Ilha Solteira/SP), SPAR (Araçatuba/SP), OURI (Ourinhos/SP), PPTE (Presidente Prudente/ SP), SJRP (São José do Rio Preto/SP) e ROSA (Rosana/SP) da RBMC do IBGE de 1 à 12 de fevereiro de 2010. O comprimento médio das 
linhas-base da rede é de $200 \mathrm{~km}$. De acordo com IBGE (2008), para linhas-base maiores do que $100 \mathrm{~km}$, o tempo de rastreio mínimo em cada par de vértices ocupado simultaneamente deve ser de 4 horas. Os dados GNSS disponíveis no site do IBGE, para cada estação da RBMC, são referentes a 24 horas de rastreio, com taxa amostral de 15 segundos. Com o auxilio do software gratuito Teqc (http://www.unavco.org/software/data-processing/teqc/teqc.html), para cada arquivo de cada estação, foram gerados dois novos arquivos de dados, contendo 6 e 4 horas de rastreio. Os arquivos com 4 e 6 horas de rastreio compreendem, respectivamente, o intervalo das 8-12 horas (hora local) e 8-14 horas (hora local).

Dessa forma, os experimentos apresentados configuram três cenários de rede, mantendo a mesma configuração de vértices, mas com diferentes tempos de rastreio. O primeiro cenário com 24 horas de rastreio, o segundo com 6 horas e o terceiro com 4 horas de rastreio, de acordo com a Tabela 1 .

Tabela 1: Cenários analisados de acordo com os tempos de rastreio GNSS

\begin{tabular}{c|c}
\hline Cenário & $\begin{array}{c}\text { Tempo de } \\
\text { rastreio }\end{array}$ \\
\hline 1 & 24 horas \\
\hline 2 & 6 horas \\
\hline 3 & 4 horas \\
\hline
\end{tabular}

Vale enfatizar que o período dos dados utilizados nos experimentos, em termos de atividade solar/ionosférica, correspondeu a um período de baixa atividade solar, o que diminui a influência dos erros de segunda e terceira ordem da ionosfera sobre os resultados (os de primeira ordem são eliminados ao utilizar receptores de dupla frequência).

As estações SJRP e ROSA foram consideradas pontos de controle da rede, injuncionadas de forma absoluta no ajustamento a partir das coordenadas oficiais (SIRGAS2000) destas estações. As demais estações foram consideradas vértices desconhecidos da rede, cujas coordenadas cartesianas geocêntricas (X, Y, Z) devem ser estimadas. A Figura 1 apresenta a rede geodésica.

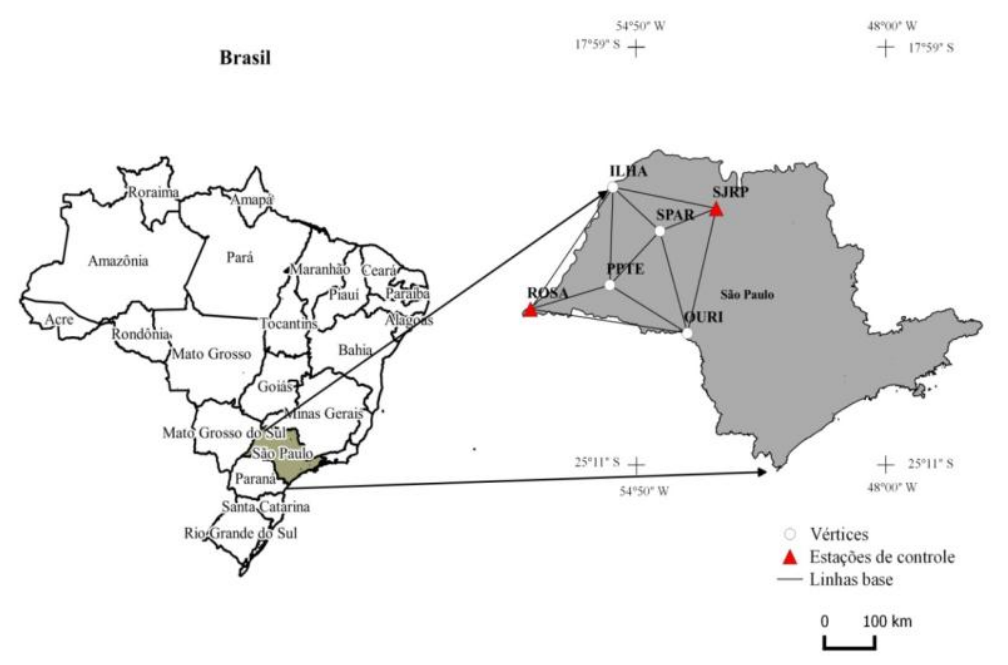

Figura 1: Configuração da rede, com onze linhas-base independentes.

Analisando a Figura 1 verifica-se que a rede é constituída de seis vértices e onze linhas-base: Bol. Ciênc. Geod., sec. Artigos, Curitiba, v. 22, no3, p.453-471, jul-set, 2016. 
PPTE-SPAR, PPTE-ILHA, PPTE-OURI, SJRP-SPAR, SJRP-ILHA, SJRP-OURI, SPARILHA, SPAR-OURI, ROSA-PPTE, OURI-ROSA, ILHA-ROSA. Nesse caso, o número de observações é igual a 33 (componentes $\Delta \mathrm{X} \Delta \mathrm{Y} \Delta \mathrm{Z}$ das onze linhas-base), o número de incógnitas é igual a 12 (coordenadas cartesianas geocêntricas dos vértices desconhecidos ILHA, SPAR, OURI, PPTE) e o número de injunções absolutas é igual a 6 (coordenadas cartesianas geocêntricas dos pontos de controle SJRP e ROSA).

A rede foi planejada contendo seis vértices que são estações pertencentes à RBMC, supracitadas. As linhas-base foram planejadas para serem independentes, e com dados GNSS correspondentes à mesma linha-base com rastreio simultâneo, ou seja, as estações que formam cada linha-base foram rastreadas simultaneamente. Após o planejamento da rede e obtenção dos dados GNSS no site do IBGE, os mesmos foram processados pelo método de PPP no serviço IBGE-PPP. Com o processamento dos dados GNSS pelo método do PPP foram obtidas as coordenadas cartesianas geocêntricas $(\mathrm{X}, \mathrm{Y}, \mathrm{Z})$, bem como os desvios-padrão e correlações entre as coordenadas, para cada vértice.

A MVC de cada vértice da rede foi obtida a partir dos resultados do PPP de cada vértice. Para formar as componentes das linhas-base $(\Delta \mathrm{X}, \Delta \mathrm{Y}, \Delta \mathrm{Z})$, a diferença entre as coordenadas obtidas pelo PPP foi realizada entre dois vértices que compunham cada linha-base. A MVC de cada linha-base foi obtida por meio da aplicação da Lei de Propagação Variância e Covariância (seguindo o método apresentado na seção 3). Com as linhas-base obtidas pelas diferenças das coordenadas entre dois vértices de cada uma das linhas-base e as respectivas MVC de cada linhabase determinadas procedeu-se o ajustamento da rede. A solução do ajustamento foi realizada pelo método paramétrico. Após o ajustamento da rede GNSS, o teste global foi aplicado e o procedimento de teste DS, e as análises de confiabilidade foram também realizadas.

\section{Resultados e Análises}

Os resultados são analisados considerando: os erros de fechamento dos circuitos fechados da rede, testes estatísticos (teste global e DS), MVC das coordenadas dos vértices da rede e os elementos de confiabilidade - confiabilidade interna (MDB), número de redundância, confiabilidade externa e razão tendência-ruído (BNR). Além disso, também são realizadas comparações desses resultados com relação ao tempo de rastreio GNSS.

\subsection{Análise de fechamento linear de circuito fechado}

Para a verificação dos erros de fechamento dos circuitos fechados, as componentes $\Delta \mathrm{X}$ de cada linha-base foram somadas, procedimento repetido para $\Delta \mathrm{Y}$ e $\Delta \mathrm{Z}$, de todas as linhas-base que compunham cada circuito das redes com os três cenários. A Tabela 2 apresenta o erro de fechamento linear (em ppm - partes por milhão) para cada circuito e para cada cenário $(4,6$ e 24 horas). 
Tabela 2: Erro de fechamento linear da rede (em ppm) em cinco circuitos fechados para os três cenários de rede (tempos de rastreio de 4, 6 e 24 horas).

\begin{tabular}{|c|c|c|c|c|}
\hline \multicolumn{5}{|c|}{ Erro de fechamento (ppm) } \\
\hline & \multirow[b]{2}{*}{ Circuito fechado } & \multicolumn{3}{|c|}{ Tempo de rastreio GNSS } \\
\hline & & 4 horas & 6 horas & 24 horas \\
\hline 1 & ROSA-ILHA-SJRP-OURI-ROSA & 0,013 & 0,024 & 0,011 \\
\hline 2 & PPTE-ILHA-SPAR-OURI-PPTE & 0,011 & 0,015 & 0,048 \\
\hline 3 & ROSA-ILHA-SPAR-PPTE-ROSA & 0,024 & 0,012 & 0,014 \\
\hline 4 & ROSA-PPTE-SPAR-SJRP-OURI-ROSA & 0,040 & 0,047 & 0,010 \\
\hline 5 & PPTE-SPAR-SJRP-OURI-PPTE & 0,061 & 0,048 & 0,016 \\
\hline
\end{tabular}

Analisando a Tabela 2 verifica-se que o menor erro de fechamento foi para o circuito 4 com 24 horas de rastreio, de $0,010 \mathrm{ppm}$. O maior erro de fechamento foi de $0,061 \mathrm{ppm}$ para o circuito 5 com 4 horas de rastreio. Em geral, os resultados de erro de fechamento de circuito foram menores nos tempos de rastreio de 24 horas quando comparados aos de 6 e 4 horas. Exceto para o segundo circuito, cujo erro de fechamento foi cerca de quatro vezes maior que para o resultado obtido em 4 horas. O teste de hipóteses foi realizado e as diferenças encontradas entre os resultados de erros de fechamento de circuito para os três tempos de rastreio analisados não é significativa (com nível de significância de 0,05).

\subsection{Análise de fechamento linear de circuito fechado}

Após a aplicação da metodologia proposta de desenvolvimento de rede GNSS a partir dos resultados obtidos pelo PPP (para os diferentes tempos de rastreio) e ajustamento da rede foram obtidas as coordenadas cartesianas geocêntricas, em SIRGAS2000, e respectivos desvios-padrão dos vértices PPTE, SPAR, ILHA e OURI. Os valores das precisões das coordenadas foram obtidos a partir da MVC dos parâmetros do ajustamento e são apresentados na figura

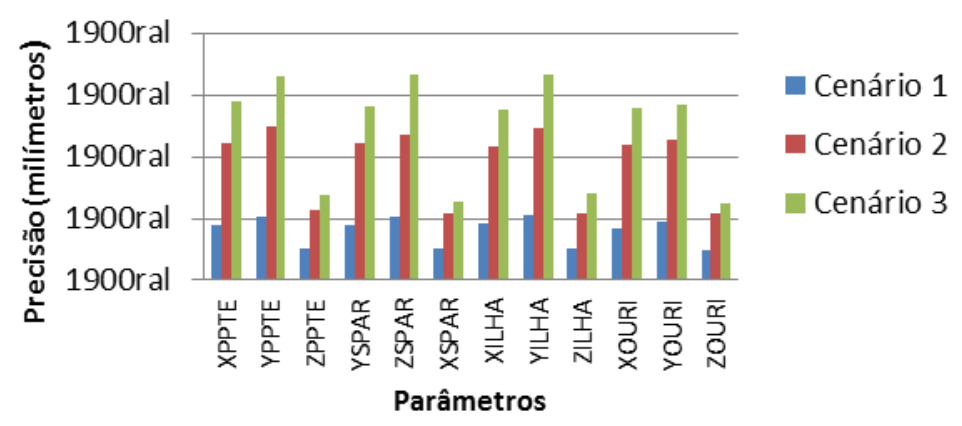

Figura 2: Desvios-padrão (em $\mathbf{m m})$ dos vértices da rede para os três cenários. 
Em média a precisão obtida para as coordenadas cartesianas geocêntricas no ajustamento foi de 4 mm para o tempo de rastreio de 24 horas; $9,5 \mathrm{~mm}$ para 6 horas e $12 \mathrm{~mm}$ para 4 horas. A precisão obtida nos vértices da rede é cerca de 3 vezes menor para o tempo de rastreio de 4 horas com relação a 24 horas; e de 2,3 vezes menor para 6 horas com relação aos resultados para 24 horas, em média. Após o ajustamento aplicaram-se os testes global do ajustamento e o DS, em cada cenário.

\subsection{Testes estatísticos}

Depois de realizado o ajustamento da rede, o teste global do ajustamento é aplicado para a detecção de erros grosseiros e de erros no modelo, em cada um dos cenários. O nível de significância do teste global foi obtido em função do nível de significância adotado para o procedimento DS $(\alpha 0=0,001)$ garantindo o mesmo poder do teste de $80 \%(\gamma=0,8)$. Sendo 21 graus de liberdade, o valor teórico para a distribuição qui-quadrado $\square 2$ a um nível de significância de 0,033 , é dado por 34,38. A Tabela 3 apresenta os valores da estatística calculada para o teste global nos três cenários e os respectivos valores de variância a posteriori obtidos.

Tabela 3: Estatísticas calculadas para o teste global nos três cenários.

\begin{tabular}{c|c|c}
\hline Cenário & Teste Global & Fator de variância a posteriori \\
\hline $\mathbf{1}$ & 21,94 & 1,04 \\
\hline $\mathbf{2}$ & 5,97 & 0,28 \\
\hline $\mathbf{3}$ & 2,66 & 0,13 \\
\hline
\end{tabular}

Analisando na Tabela 3 verifica-se que a estatística de teste global calculada resulta em 21,94 para o primeiro cenário ( 24 horas), 5,97 para o segundo cenário (6 horas) e 2,66 para o terceiro cenário (4 horas). Dessa forma, como a estatística calculada é menor que o valor crítico teórico, em todos os três cenários, a hipótese nula do teste global do ajustamento não é rejeitada ao nível de significância de 0,033 e o ajustamento é aceito pelo teste global, em todos os cenários. Como o ajustamento não foi rejeitado pelo teste global, seguindo o procedimento do método de Baarda (1968) não é necessário aplicar o teste DS. Porém, apenas para confirmação, aplicou-se o DS e de fato nenhuma observação foi identificada com nível de significância de 0,001 , nos três cenários.

Ainda analisando a Tabela 3 os valores de fator de variância a posteriori, para 21 graus de liberdade, verifica-se para o primeiro cenário o valor 1,04 demonstrando que nesse caso a precisão das observações está adequada. No segundo e terceiro cenários o fator de variância a posteriori foi, respectivamente, de 0,28 e 0,13 . Ou seja, menor que para o primeiro cenário, além do valor do terceiro cenário ser menor que do segundo. $O$ fator de variância a posteriori nesses casos demonstra que a precisão das observações é menos realística que no primeiro cenário, e a precisão dos parâmetros (coordenadas dos vértices da rede) pode ser melhor que as já obtidas no ajustamento. 


\subsection{Análise de Confiabilidade}

As medidas de confiabilidade convencionais foram obtidas considerando um poder de teste de $80 \%$ e nível de significância de 0,001 , o que implica em um parâmetro de não centralidade de 17,075. A Tabela 4 apresenta os valores máximos, mínimos e médios de confiabilidade interna (MDB) obtidos para os três cenários.

Tabela 4: Valores máximos, mínimos e médios de MDB em cada cenário.

\begin{tabular}{c|c|c|c}
\hline \multirow{2}{*}{ Cenário } & \multicolumn{3}{|c}{ MDB (centímetros) } \\
\cline { 2 - 4 } & Máximo & Mínimo & Médio \\
\hline $\mathbf{1}$ & 2,9 & 0,9 & 1,97 \\
\hline $\mathbf{2}$ & 10,4 & 2,41 & 5,26 \\
\hline $\mathbf{3}$ & 19,63 & 3,46 & 7,76 \\
\hline
\end{tabular}

Analisando a Tabela 4 verifica-se que o MDB máximo encontrado para o terceiro cenário é cerca de 7 vezes maior que para o primeiro. E o segundo é cerca de 3,6 vezes maior que o primeiro, também com relação ao valor máximo de confiabilidade interna. Com relação aos valores mínimos de MDB encontrados em cada cenário verifica-se que o segundo e o terceiro cenários, respectivamente, são cerca de 3,9 e 2,7 vezes maiores que os do primeiro cenário. Analisando os valores médios de MDB nota-se aproximadamente a mesma proporção entre os cenários obtida pra os valores mínimos de MDB. Assim, com maior tempo de rastreio verifica-se melhora na confiabilidade interna da rede. A Tabela 5 apresenta os valores máximos, mínimos e médios de número de redundância encontrados para os três cenários.

Tabela 5: Valores máximos, mínimos e médios de número de redundância em cada cenário.

\begin{tabular}{c|c|c|c}
\hline \multirow{2}{*}{ Cenário } & \multicolumn{3}{|c}{ Redundância } \\
\cline { 2 - 4 } & Máximo & Mínimo & Médio \\
\hline $\mathbf{1}$ & 0,73 & 0,56 & 0,64 \\
\hline $\mathbf{2}$ & 0,91 & 0,52 & 0,64 \\
\hline $\mathbf{3}$ & 0,83 & 0,51 & 0,64 \\
\hline
\end{tabular}

Na Tabela 5 verifica-se que a média de número de redundância nos três cenários foi o mesmo, de 0,64. Além disso, os valores mínimos encontrados em cada cenário são aproximadamente de mesma magnitude e maiores que 0,5 . Com relação aos valores máximos de redundância, o maior valor encontrado é para o cenário intermediário, de 6 horas de rastreio. No primeiro cenário, com maior tempo de rastreio verifica-se mais homogeneidade nos valores, sendo a média igual a dos demais cenários. A Tabela 6 apresenta os valores máximos, mínimos e médios de razão tendência-ruído (BNR) encontrados para os três cenários.

Tabela 6: Valores máximos, mínimos e médios de BNR em cada cenário.

\begin{tabular}{c|c|c|c}
\hline \multirow{2}{*}{ Cenário } & \multicolumn{3}{|c}{ BNR (metros) } \\
\cline { 2 - 4 } & Máximo & Mínimo & Médio \\
\hline $\mathbf{1}$ & 12,86 & 7,43 & 9,91 \\
\hline $\mathbf{2}$ & 15,58 & 1,91 & 10,43 \\
\hline $\mathbf{3}$ & 23,02 & 4,37 & 12,65 \\
\hline
\end{tabular}

Bol. Ciênc. Geod., sec. Artigos, Curitiba, v. 22, no3, p.453-471, jul-set, 2016. 
Ao analisar a Tabela 6 verifica-se que a média de BNR é menor para o primeiro cenário, ou seja, o maior tempo de rastreio melhorou a confiabilidade externa da rede. O menor valor de BNR mínimo é para o cenário 2. Com relação aos valores máximos se verifica maior BNR para o cenário3, com menor tempo de rastreio, sendo cerca de 1,8 vezes maior que no primeiro.

Para cada cenário foram realizadas análises dos parâmetros de confiabilidade conjuntamente, relacionando os maiores, menores e valores intermediários de MDB com relação à confiabilidade externa da rede.

\subsection{Cenário 1}

A componente Z da linha-base ROSA_OURI apresenta o maior valor $(0,727)$ de redundância na rede. Essa componente da linha-base ROSA_OURI também apresenta o menor valor para o MDB, de 9 milímetros. As componentes X da linha-base OURI_SPAR possuem maior valor de BNR (12,86 metros). A Tabela 7 apresenta as confiabilidades externas relativas à observação com maior MDB, MDB intermediário e menor MDB. O maior valor de MDB encontrado é de 2,9 centímetros na observação $\triangle$ XILHA_SPAR, o MDB intermediário é de 2,15 centímetros (média dos valores de MDB é 1,97 centímetros) na observação $\triangle$ YROSA_OURI e menor MDB de 9 milímetros na observação $\triangle$ ZROSA_OURI.

$\mathrm{Na}$ análise conjunta das medidas de confiabilidade nota-se que o maior MDB encontrado, de 2,9 centímetros quando não detectado causa um erro de apenas 6 milímetros na componente XILHA. Verifica-se também que o MDB intermediário de 2,15 centímetros quando não detectado causa um erro de 7 milímetros na coordenada YOURI. Ainda nesse sentido, analisando a influência do menor MDB, de 9 milímetros, quando não detectado causa um erro de 2 milímetros na coordenada ZOURI, sendo o número de redundância nesse caso de 0,727 , o maior valor para a rede. O valor máximo de confiabilidade externa nesse cenário foi de 9 milímetros.

Tabela 7: Confiabilidade externa das observações com maior, menor e valor intermediário de MDB no cenário 1.

\begin{tabular}{c|c|c|c}
\hline & \multicolumn{3}{|c}{$\begin{array}{c}\text { Confiabilidade Externa das Observações (em } \\
\text { metros) }\end{array}$} \\
\hline Parâmetros & $\begin{array}{l}\Delta \mathrm{X}_{\text {ILHA SPAR- }} \\
\text { Maior MDB }\end{array}$ & $\begin{array}{l}\Delta \text { Y }_{\text {ROSA OURI- }} \\
\text { MDB Inter. }\end{array}$ & $\begin{array}{l}\Delta Z_{\text {ROSA OURI- }} \\
\text { Menor MDB }\end{array}$ \\
\hline X_PPTE & $-0,001$ & 0,000 & 0,000 \\
\hline Y_PPTE & 0,000 & $-0,003$ & 0,000 \\
\hline Z_PPTE & 0,000 & 0,000 & 0,001 \\
\hline X_SPAR & $-0,005$ & 0,000 & 0,001 \\
\hline Y_SPAR & 0,000 & $-0,003$ & 0,001 \\
\hline Z_SPAR & 0,000 & 0,000 & 0,001 \\
\hline X_ILHA & 0,006 & 0,000 & 0,000 \\
\hline Y_ILHA & 0,000 & $-0,002$ & 0,000 \\
\hline Z_ILHA & 0,000 & 0,000 & 0,000 \\
\hline X_OURI & $-0,001$ & 0,001 & 0,001 \\
\hline Y_OURI & 0,000 & $-0,007$ & 0,001 \\
\hline Z_OURI & 0,000 & 0,000 & 0,002 \\
\hline
\end{tabular}

Bol. Ciênc. Geod., sec. Artigos, Curitiba, v. 22, noำ, p.453-471, jul-set, 2016. 


\subsection{Cenário 2}

A componente X da linha-base SJRP_OURI apresenta o maior valor $(0,914)$ de redundância na rede e também apresentou a menor confiabilidade interna (maior valor para o MDB) de 10,4 centímetros. Mas, ao contrário do primeiro cenário, essa observação possui o menor valor de BNR da rede, de 1,905. As componentes X da linha-base OURI_PPTE possui maior valor de BNR (15,58 metros). Dentre todas as linhas-base, a OURI_PPTE provoca o maior efeito nos parâmetros em decorrência de erros não detectados nas suas observações, sendo seus valores de números de redundância (em média de 0,55 ) abaixo da média para a rede $(0,64)$ e valores de MDB baixos.

A Tabela 8 apresenta apenas três confiabilidades externas, relativas à observação com maior MDB, MDB intermediário e menor MDB. O maior valor de MDB encontrado é de 10,4centímetros na observação $\triangle$ XSJRP_OURI, o MDB intermediário é de 5,22 centímetros (média dos valores de MDB é 5,26 centímetros) na observação $\triangle$ YSPAR_SJRP e menor MDB de 2,41centímetros na observação $\triangle$ ZSPAR_SJRP.

Analisando as medidas de confiabilidade conjuntamente verifica-se que o maior MDB encontrado, de 10,4 centímetros quando não detectado causa um erro de apenas 9 milímetros na coordenada XOURI. O que é coerente, considerando também o número de redundância local desta observação que é de 0,914 (maior valor de redundância na rede neste cenário). Ou seja, $91 \%$ do erro é refletido no resíduo desta observação e $9 \%$ é absorvido na estimação dos parâmetros incógnitos).

Tabela 8: Confiabilidade externa das observações com maior, menor e valor intermediário de MDB no cenário 2.

\begin{tabular}{l|c|c|c}
\hline & \multicolumn{3}{|c}{$\begin{array}{c}\text { Confiabilidade Externa das Observações (em } \\
\text { metros) }\end{array}$} \\
\hline Parâmetros & $\begin{array}{c}\Delta \mathrm{X}_{\text {SJRP OURI- }} \\
\text { Maior MDB }\end{array}$ & $\begin{array}{l}\Delta \text { Y }_{\text {SPAR SJRP- }} \\
\text { MDB Inter. }\end{array}$ & $\begin{array}{c}\Delta \text { Z }_{\text {SPAR SJRP- }} \\
\text { Menor MDB }\end{array}$ \\
\hline X_PPTE & $-0,004$ & $-0,001$ & 0,001 \\
\hline Y_PPTE & $-0,001$ & 0,010 & 0,000 \\
\hline Z_PPTE & 0,000 & 0,000 & 0,004 \\
\hline X_SPAR & $-0,004$ & $-0,001$ & 0,001 \\
\hline Y_SPAR & $-0,001$ & 0,021 & 0,000 \\
\hline Z_SPAR & 0,000 & 0,000 & 0,009 \\
\hline X_ILHA & $-0,002$ & 0,000 & 0,000 \\
\hline Y_ILHA & 0,000 & 0,007 & 0,000 \\
\hline Z_ILHA & 0,000 & 0,000 & 0,003 \\
\hline X_OURI & $-0,009$ & 0,000 & 0,001 \\
\hline Y_OURI & $-0,002$ & 0,009 & $-0,001$ \\
\hline Z_OURI & $-0,001$ & 0,000 & 0,004 \\
\hline
\end{tabular}


Verifica-se também que o MDB intermediário de 5,22 centímetros quando não detectado causa um erro de 2,1 centímetros na coordenada YSPAR. Ainda nesse sentido, analisando a influência do menor MDB, de 2,41 centímetros quando não detectado causa um erro de 9milímetros na coordenada ZSPAR. Isso faz sentido, considerando também o número de redundância local desta observação que é de 0,61 .

\subsection{Cenário 3}

As componentes $\mathrm{Z}$ e $\mathrm{Y}$ da linha-base OURI-SPAR apresentaram o maior valor $(0,83)$ e o terceiro maior valor $(0,82)$ de redundância, respectivamente, na rede e a componente $Y$ dessa linha-base apresentou a menor confiabilidade interna (maiores valores para o MDB) de 19,63 centímetros. As componentes $\mathrm{Z}$ e Y da linha-base OURI-SPAR também são as que possuem maiores valores de BNR (23 e 22,3 metros, respectivamente). Isto significa que entre todas as linhas-base, a OURI-SPAR provoca o maior efeito nos parâmetros em decorrência de erros não detectados nas suas observações, mesmo os seus números de redundância sendo maiores para a rede. Isso pode ser devido ao fato de o MDB dessas observações ser cerca de 2,5 vezes maior que a média dos valores de MDB da rede.

Como a rede geodésica em estudo possui 33 observações, pela teoria de confiabilidade convencional, tem-se 33 confiabilidades externas associadas (uma para cada observação). A Tabela 9 apresenta apenas três destas confiabilidades externas, relativas à observação com maior MDB, MDB intermediário e menor MDB.

Tabela 9: Confiabilidade externa das observações com maior, menor e valor intermediário de MDB no cenário 3.

\begin{tabular}{|c|c|c|c|}
\hline \multirow[b]{2}{*}{ Parâmetros } & \multicolumn{3}{|c|}{$\begin{array}{c}\text { Confiabilidade Externa das Observações (em } \\
\text { metros) }\end{array}$} \\
\hline & \begin{tabular}{|l|}
$\mathrm{Y}_{\text {OURI SPAR }}$ \\
Maior MDB
\end{tabular} & \begin{tabular}{|l|}
$\Delta \mathrm{X}_{\text {SJRP ILHA }}$ \\
MDB Inter.
\end{tabular} & \begin{tabular}{|l|} 
\\
$Z_{\text {PPTE ROSA }}$ \\
Menor MDB
\end{tabular} \\
\hline X_PPTE & 0,002 & $-0,003$ & 0,004 \\
\hline Y_PPTE & $-0,003$ & $-0,007$ & $-0,004$ \\
\hline Z_PPTE & $-0,002$ & 0,001 & 0,012 \\
\hline X_SPAR & 0,013 & $-0,002$ & 0,002 \\
\hline Y_SPAR & $-0,020$ & $-0,009$ & $-0,002$ \\
\hline Z_SPAR & $-0,016$ & 0,001 & 0,006 \\
\hline X_ILHA & 0,004 & 0,000 & 0,001 \\
\hline Y_ILHA & $-0,006$ & $-0,023$ & 0,000 \\
\hline Z_ILHA & $-0,005$ & 0,001 & 0,004 \\
\hline X_OURI & $-0,013$ & $-0,001$ & 0,002 \\
\hline Y_OURI & 0,016 & $-0,003$ & $-0,001$ \\
\hline Z_OURI & 0,014 & 0,000 & 0,004 \\
\hline
\end{tabular}

O maior valor de MDB encontrado é de 19,93 centímetros na observação $\triangle$ YOURI_SPAR, o MDB intermediário é de 7,62 centímetros (média dos valores de MDB é 7,73 centímetros) na 
observação $\triangle$ XSJRP_ILHA e menor MDB de 3,46 centímetros na observação $\triangle$ ZPPTE ROSA. Analisando essas medidas de confiabilidade conjuntamente verifica-se que o maior MDB encontrado, de 19,93 centímetros, correspondente a observação $\triangle$ YOURI_SPAR, quando não detectado causa um erro de 2 centímetros na coordenada YSPAR. Fato este que é pertinente, considerando também o número de redundância local desta observação que é de 0,82 (ou seja, $82 \%$ do erro é refletido no resíduo desta observação e $18 \%$ é absorvido na estimação dos parâmetros incógnitos).

Também se verifica que o MDB intermediário de 7,7 centímetros, correspondente à observação $\triangle X S J R P$ ILHA quando não detectado causa um erro de 2,3 centímetros na mesma coordenada YILHA. Ainda nesse sentido, analisando a influência do menor MDB, de 3,5 centímetros, correspondente a observação $\triangle$ ZPPTE_ROSA, se não detectado causa um erro de 1,2 centímetros na coordenada ZPPTE, o que faz sentido considerando também o número de redundância local desta observação que é de 0,64.

\section{Considerações e Conclusões}

O foco principal deste artigo foi apresentar, aplicar e avaliar uma proposta de método de desenvolvimento de rede utilizando dados GNSS processados por PPP. O método foi aplicado em três cenários de rede geodésica, variando os tempos de rastreio GNSS nos vértices. O primeiro cenário com 24 horas de rastreio, o segundo com 6 horas e o terceiro com 4 horas de rastreio. A rede em questão possui seis vértices, sendo dois deles injuncionados, resultando em 33 observações e 12 parâmetros incógnitos.

Os dados GNSS de cada cenário e de cada vértice foram processados pelo método de PPP no serviço IBGE-PPP. As componentes $\Delta \mathrm{X}, \Delta \mathrm{Y}$ e $\Delta \mathrm{Z}$ de todas as linhas-base foram determinadas a partir dos resultados do PPP. A MVC das linhas-base foi obtida por meio da aplicação da Lei de Propagação Variância e Covariância. Com as linhas-base e suas respectivas MVC determinadas foi realizado o ajustamento da rede através do MMQ utilizando o método paramétrico.

Antes do ajustamento foi realizada uma pré-análise da rede, com a verificação dos erros de fechamento de circuitos fechados em cada cenário. Em geral, os resultados de erro de fechamento de circuito foram menores nos tempos de rastreio de 24 horas quando comparados aos de 6 e 4 horas, sendo da ordem de 0,02 ppm. O teste de hipóteses foi realizado e as diferenças encontradas entre os resultados de erros de fechamento de circuito para os tempos de rastreio analisados não é significativa (com nível de significância de 0,05).

Após a realização do ajustamento da rede para os três cenários, o teste global foi aplicado, sendo o ajustamento dos três cenários aceitos, com nível de significância de 0,033. A partir da análise do fator de variância a posteriori verificou-se que no primeiro cenário, com 24 horas de rastreio, a precisão das observações foi mais realística que com relação aos cenários com 6 e 4 horas de rastreio. Nesses outros dois cenários a precisão pode ser melhor do que a estimada, o que mostra que o resultado do PPP para 6 e 4 horas de rastreio pode estar sendo mais conservador do que a real precisão (o que não gera um problema - na verdade, o contrário seria complicado). Mesmo tendo o ajustamento passado no teste global, o teste DS também foi aplicado e nenhuma observação identificada, conforme o esperado, com nível de significância de 0,001 , nos três cenários. Os valores de desvios-padrão para as coordenadas obtidas a partir da MVC dos parâmetros do ajustamento mostram que a precisão dos vértices da rede é cerca de 3 vezes menor

Bol. Ciênc. Geod., sec. Artigos, Curitiba, v. 22, no3, p.453-471, jul-set, 2016. 
para o tempo de rastreio de 4 horas com relação a 24 horas e de 2,3 vezes menor para 6 horas com relação aos resultados para 24 horas, em média.

Analisando a confiabilidade interna da rede para os três cenários verifica-se que o MDB máximo encontrado para o terceiro cenário é cerca de 7 vezes maior que para o primeiro. E o segundo cenário é cerca de 3,6 vezes maior que o primeiro, também com relação ao valor máximo de confiabilidade interna. Com relação aos valores mínimos de MDB encontrados em cada cenário conclui-se que o segundo e o terceiro cenários, respectivamente, são cerca de 3,9 e 2,7 vezes maiores que o primeiro. Analisando os valores médios de MDB se verifica aproximadamente a mesma proporção entre os cenários obtida para os valores mínimos de MDB.

Com relação à redundância da rede verificou-se que a média de número de redundância nos três cenários foi o mesmo, de 0,63 e os valores mínimos encontrados em cada cenário são aproximadamente de mesma magnitude e maiores que 0,5 . Com relação aos valores máximos de redundância, o maior valor encontrado é para o cenário intermediário, de 6 horas de rastreio. No primeiro cenário, com maior tempo de rastreio verifica-se mais homogeneidade nos valores, sendo a média igual aos demais cenários.

No terceiro cenário os valores máximos e mínimos de confiabilidade externa foram, respectivamente, 4,8 e 0,8 centímetros. No segundo cenários os valores máximo e mínimo foram de 2,7 e 0,6 centímetros, respectivamente. Enquanto que no primeiro cenário, com 24 horas de rastreio, os valores de confiabilidade externa se encontraram entre 0,9 e 0,2 centímetros.

A vantagem do método de rede GNSS utilizando o PPP com relação a somente o PPP para os pontos GNSS individualmente é a possibilidade de ajustar a rede e, a partir dos resultados do ajustamento, a possibilidade de aplicação de controle de qualidade da mesma, conforme apresentados nesse artigo.

O experimento foi realizado com dados de um período de baixa atividade solar, sendo interessante aplicar o método proposto para um período de alta atividade solar, sendo recomendado para trabalhos futuros.

Para trabalhos futuros também pretende-se comparar os resultados de uma rede a partir de um posicionamento relativo e a partir de um PPP de acordo com a metodologia apresentada nesse artigo.

\section{AGRADECIMENTOS}

Ao CNPq (Bolsa de Mestrado 2013-2015; Bolsa Produtividade em Pesquisa proc. 303306/20122; Projeto Universal 14/2012 proc. 477914/2012-8). Ao IBGE pelos dados da RBMC e pela disponibilização do serviço do PPP-on-line;

\section{REFERÊNCIAS BIBLIOGRÁFICAS}

Baarda, W. A testing procedure for use in geodetic networks. Publications on Geodesy, New Series, v. 2, n. 5, Delft: Netherlands Geodetic Commission, 1968.

El-Mowafy, A. Analysis of web-based GNSS post-processing services for static and kinematic positioning using short data spans. Survey Review, v.43, n.323,p. 535-549, 2011. 
Faustino, R. C. Posicionamento Por Ponto Preciso estático e cinemático. 2006. 105f. Dissertação (Mestrado em Ciências Cartográficas) - Faculdade de Ciências e Tecnologia, Universidade Estadual Paulista, Presidente Prudente. 2006.

Gao, Y and Shen, X. A New Method for Carrier Phase Based Precise Point Positioning. Navigation, Journal of the Institute of Navigation, Vol. 49, No. 2, 2002.

Gemael, C. Introdução ao ajustamento de observações: aplicações geodésicas. Curitiba: Editora da UFPR, 1994. 319p.

Ghilani, C. D.; Wolf, P. R. Adjustment Computations: Spatial Data Analysis. 4. ed. New York: John Wiley \& Sons, 2006. 611p.

Hofmann-Wellenhof, B.; Lichtengger, H.; Wasle, E. 2008. GNSS - Global Navigation Satellite System: GPS, GLONASS, GALILEO, and More. New York, Springer Wien, 516 p.

IBGE. 2008. Recomendações para Levantamentos Relativos Estáticos - GPS. Disponível em: <ftp://geoftp.ibge.gov.br/documentos/geodesia/pdf/recom_gps_internet.pdf>.

IBGE. 2013. Manual do Usuário Posicionamento Por Ponto Preciso. Diretoria de Geociências. Disponível em: http://www.ibge.gov.br/home/geociencias/geodesia/ppp/manual_ppp.pdf.

Kavouras, M. On the Detection of Outliers and the Determination of Reliability in Geodetic Networks. 1982. M. Sc. E. Thesis - Department of Geodesy and Geomatics Engineering, University of New Brunswick, Fredericton, Canada, 1982.

Klein, I.; Matsuoka, M. T.; Souza, S. F.: Análise do serviço on-line de PPP (GDGPS - APPS) para Receptores de Dupla Freqüência: um estudo envolvendo dados de estações da RBMC, in: III Simpósio Brasileiro de Ciências Geodésicas e Tecnologias da Geoinformação, Recife - PE, 27-30 de julho de 2010,p. 001-007.

Klein, I. Controle de Qualidade no Ajustamento de Observações Geodésicas. Dissertação (Mestrado) - Programa de Pós-Graduação em Sensoriamento Remoto, UFRGS. 2012.

Kouba, J.; Heroux, P. Precise Point Positioning using IGS orbit and clock products. GPS Solutions, v. 5, n. 2, p. 12-28, 2001.

Leick, A. 2004.GPS Satellite Surveying, 3a ed., New Jersey, John Wiley \& Sons, 435p.

Machado, W. C.; Monico, J. F. G. Controle de Qualidade do Ajustamento Recursivo de Observações GPS em Linhas de Base Curtas. Boletim de Ciências Geodésicas, v10, n2. Curitiba: Universidade Federal do Paraná (UFPR), 2004. p 123-140

Marques, H. A.; Monico, J. F. Galera ; Shimabukuro, M. H. ; Oyama, R. T. ; Wentz, J. P. T. G. . PPP em tempo real: fundamentos, implementação computacional e analises de resultados no modo estático e cinemático. RBC. Revista Brasileira de Cartografia (Online), v. 66, p. 13311345, 2014.

Matsuoka, M.T.; Azambuja, J.L.F.; Souza, S.F. Potencialidades do serviço on-line de Posicionamento por Ponto Preciso (CSRS-PPP) em aplicações geodésicas. Gaea - Journal of Geoscience, vol. 5, n. 1, jan/jun 2009, p. 42-49.

Monico, J.F.G. Posicionamento pelo GNSS: Descrição, fundamentos e aplicações. São Paulo: Editora Unesp, 2008, 476 p.

Ocalan, T.; Erdogan, B.; Tunalioglun. Analysis of web-based online services for GPS relative and precise point positioning techniques. Bol.Ciênc.Geod., sec. Artigos,Curitiba, vol. 19, n $^{\circ} 2$, p.191-207, abr-jun, 2013. 
Oliveira, R.; Dalmolin, Q. A Influência da Redundância da Observação sobre a Precisão dos Parâmetros. Boletim de Ciências Geodésicas, v. 14, n. 3, p. 295-315, 2008.

Perdigão, T. D.; Santos, A. P. Avaliação da acurácia a partir de diferentes tempos de rastreio utilizando a solução PPP on-line do IBGE. Anais do III Simpósio Brasileiro de Ciências Geodésicas e Tecnologias da Geoinformação. 2010.

Rocha, G. D. C.; Marques, H. A. ; Monico, J F G ; Machado, W. C. . Avaliação de modelos ionosféricos globais e regionais no Posicionamento por Ponto Preciso. In: SIMGEO - Simpósio Brasileiro de Ciências Geodésicas e Tecnologias da Geoinformação, 2014, Recife. Anais do VSIMGEO. Recife: UFPE, 2014.

Rosa, G.P.S. Análise de Séries Temporais de Coordenadas Estimadas com GPS: Uma Proposta Metodológica para Eliminação de Efeitos Sazonais.Tese (Mestrado) - Universidade Estadual Paulista,Presidente Prudente, 2008. 106 p.

Souza, A. N.; Garnes, S. J. A. ; Marques, H. A. . Avaliação do Posicionamento GNSS obtido pelo Métodos Cinemáticos RTK/NTRIP e PPP em Tempo Real. RBC. Revista Brasileira de Cartografia (Online), v. 5, p. 1117- 1133, 2014.

Teunissen, P. J. G. Testing theory: an introduction. 2. ed. Delft: Ed. VSSD, 2006. 147p.

Witchayangkoon, B. Elements of GPS precise point positioning. 2000. 265f. PhDThesis in Spatial Information Science and Engineering. University of Maine, USA. 2000.

Zumberge, J.F.; Heflin, M.B.; Jefferson,D.C.; Watkins, M.M.; Webb, F.H.1997.Precise Point Positioning for the efficient and robust analysis of GPS Data from Large Networks. Journal of Geophysical Research, 102(B3):5005-5017.http://dx.doi.org/10.1029/96JB03860.

Recebido em Agosto de 2015.

Aceito em Outubro de 2015. 